\title{
Zum Schutz der Menschenrechte durch die europäischen Gerichtshöfe ${ }^{1}$
}

\section{Prolog: Burkhard Hirsch und die Menschenwürde}

Als liberaler Politiker und hervorragender Jurist hat Burkhard Hirsch sich für die Grundund Menschenrechte besonders engagiert. Bedeutsam sind diesbezüglich seine zahlreichen umfassenden wissenschaftlichen Beiträge, die in renommierten Fachzeitschriften und - vor allem auch - in überregionalen Medien erschienen sind. Zumeist sind sie an den Grundrechten orientiert und richten sich an zwar nicht immer juristisch geschulte, aber an elementaren Freiheiten interessierte Leser. Auch seine Tätigkeit als Rechtsanwalt ist hervorzuheben, gerade sein Einsatz vor dem Bundesverfassungsgericht: Erinnert sei an das Urteil des Ersten Senats vom 3. März $2004^{2}$ betreffend den Lauschangriff bzw. die akustische Wohnraumüberwachung. Das Gericht hat diese Maßnahmen mit der Unantastbarkeit der Menschenwürde zwar als vereinbar angesehen, aber die Durchführungsvorschriften in der Strafprozessordnung teilweise für verfassungswidrig erklärt. Hervorgehoben sei hier auch die abweichende Meinung, die meine Kollegin, Richterin Renate Jäger mit Richterin Christine Hohmann-Dennhardt, verfasst hat ${ }^{3}$.

In diesem Zusammenhang ist ferner auf das Urteil des ersten Senats vom 15. Februar $2006^{4}$ hinzuweisen. Hier hat sich Hirsch ebenfalls verdient gemacht und die Nichtigkeit des Luftsicherheitsgesetzes bewirkt. Das Gericht hat entschieden, dass der Abschuss von zur Tötung von Menschen eingesetzten Flugzeugen das Recht auf Leben und Menschenwürde der tatunbeteiligten Menschen an Bord verletzt. Burkhard Hirsch liefert den Beweis, dass Politik und Juristerei nicht unvereinbar sind und sich im Sinne der Menschenrechte ergänzen.

Einer Anfrage des Laudators war als Anhang der Vortrag von Burkhard Hirsch beigefügt, den er selbst nicht so sehr als »Rede«, sondern als einen »politischen Stoßseufzer« bezeichnet. Die Einladung wurde folgendermaßen ergänzt: »Der Fachbereich Rechtswissenschaft freut sich auf Ihre Erwiderung (sei es Zurückweisung oder Mitseufzer).« Ich habe erwidert, dass ich nach eingehendem Studium des scharfsinnigen Beitrags von Burkhard Hirsch weder in der Lage bin »mitzuseufzen« - ich bin eigentlich immer noch Optimist - noch diesen Beitrag »zurückzuweisen«. Im Gegenteil, ich möchte in meiner kurzen Erwiderung die »Bemerkungen zum Schutz der Grundrechte im >Raum der Freiheit, der Sicherheit und des Rechts der Europäischen Gemein$s c h a f t \ll$ konstruktiv ergänzen, wenngleich dieses Unterfangen wegen der Komplexität des Referates von Hirsch schwierig ist.

1 Der Autor ist Richter am Europäischen Gerichtshof für Menschenrechte. Er hielt dieses CoReferat anlässlich der Verleihung der Ehrendoktorwürde an Dr. Burkhard Hirsch durch den Fachbereich Rechtswissenschaft der Johann Wolfgang Goethe-Universität, Frankfurt am Main am 17. November 2006.

2 BVerfGE 109, 279; EuGRZ, 2004, 163.

3 BVerfGE 109, 382; EuGRZ, 2004, 193.

41 BvR 357/05; EuGRZ, 2006, 83. 
Der Autor hat auf den Schutz der Grundrechte in der Solange I-Entscheidung ${ }^{5}$ und auf das seitdem veränderte Bild hingewiesen. Er ermuntert das Bundesverfassungsgericht - im Hinblick auf das von ihm aufgezeigte demokratische Defizit in der Europäischen Union -, seine Rechtsprechung über die unverzichtbare und unmittelbare Anwendung des Rechts auf Menschenwürde gemäß Art. 1 des Grundgesetzes auch gegenüber den Europäischen Institutionen und dem EuGH durchzusetzen. Das Bundesverfassungsgericht solle selbst darüber entscheiden, ob dieser für unser Rechtsdenken unverzichtbare Anspruch auf Wahrung der Menschenwürde in einem konkreten Einzelfall verletzt wurde oder nicht. Hirsch erinnert daran, dass geltendes Recht der Europäischen Gemeinschaft (EG) oder der Europäischen Union (EU) gegebenenfalls in Konflikt mit den Grund- und Menschenrechten geraten kann. In diesem Kontext erwähnt er im Besonderen folgende Punkte:

- Er bedauert, dass die EU immer noch nicht Mitglied der Europäischen Menschenrechtskonvention ist.

- Er weist unter Bezug auf das Matthews-Urteil vom 18. Februar $1999^{6}$ darauf hin, dass der Europäische Gerichtshof für Menschenrechte (EGMR) allerdings indirekt das Recht der EU prüfen kann.

- Burkhard Hirsch unterstreicht, dass der Europäische Gerichtshof (EuGH) gleichermaßen die Pflicht aller Gemeinschaftsorgane zur Berücksichtigung und Verwirklichung der Grund- und Menschenrechte betont, wie sie Gegenstand der allgemeinen Rechtsüberzeugungen der Mitglieder der Gemeinschaft geworden sind.

Seine pessimistische Sicht und Kritik indessen, wonach der EuGH der Effektivität von Verwaltungsentscheidungen für den Gemeinsamen Markt gegenüber dem individuellen Rechtschutz stets einen deutlichen Vorrang gegeben habe, kann ich nur bedingt teilen. Sicher, die Europäischen Grundfreiheiten im Bereich des gemeinsamen Marktes sind in ständiger Rechtsprechung vom EuGH besonders geschützt und durchgesetzt worden. Aber gerade, wenn diese Rechte des gemeinsamen Marktes in Konflikt mit den Grundund Menschenrechten geraten sind, hat der EuGH diesen letztgenannten Rechten - so scheint es mir (besonders in der jüngsten Rechtssprechung) - deutlich den Vorrang gegeben. Die Rechtssachen Schmidberger und Omega sind hier von besonderer Bedeutung.

\section{Der EuGH: Schmidberger und Omega}

Im Urteil vom 12. Juni $2003^{7}$ hat der EuGH - bezüglich einer Kollision von Grundrechten mit EG-Grundfreiheiten anlässlich einer Brenner-Blockade - festgehalten, dass keine Haftung Österreichs gegenüber einer LKW-Spedition wegen eben dieser Blockade durch Umweltschutzdemonstranten vorliegt. Das vorlegende Gericht warf in seinem Vorlagebeschluss außerdem die Frage auf, ob der im EG-Vertrag verankerte Grundsatz des freien Warenverkehrs diesen Grundrechten vorgeht.

5 BVerfGE 37, 271; EuGRZ 1974, 5.

6 RJD 1999-I.

7 Eugen Schmidberger, Internationale Transporte und Planzüge, gegen Republik Österreich, Rs. C-112/00, Slg. 2003, I-5659; EuGRZ 2003, 492. 
Der EuGH hat festgehalten, der Umstand, dass die zuständigen Behörden eines Mitgliedstaats eine Versammlung - die zu einer ununterbrochenen nahezu 30-stündigen völligen Blockade einer wichtigen Verkehrsverbindung wie der Brenner-Autobahn führte - nicht untersagten, könne eine Beeinträchtigung des innergemeinschaftlichen Warenverkehrs darstellen. Die fehlende behördliche Untersagung sei daher als Maßnahme gleicher Wirkung wie mengenmäßige Beschränkungen anzusehen, die grundsätzlich mit den gemeinschaftsrechtlichen Verpflichtungen - die sich aus den Artikeln 30 und 34 EG-Vertrag in Verbindung mit Artikel 5 EG-Vertrag ergeben - unvereinbar ist, sofern die Nichtuntersagung nicht objektiv gerechtfertigt werden kann.

Bezüglich der Rechtfertigung der Beeinträchtigung hat der EuGH hervorgehoben, dass »[79.] ...die Grundrechte, um die es im Ausgangsrechtsstreit geht, zwar ausdrücklich durch die EMRK anerkannt sind. Sie stellen wesentliche Grundlagen einer demokratischen Gesellschaft dar, doch können, wie sich aus dem Wortlaut des jeweiligen Absatzes 2 der Artikel 10 und 11 EMRK ergibt, auch die Meinungsäußerungs- und die Versammlungsfreiheit bestimmten - durch Ziele des Allgemeininteresses gerechtfertigten - Beschränkungen unterworfen werden, sofern diese Ausnahmen gesetzlich vorgesehen, von einem oder mehreren nach diesen Bestimmungen berechtigten Zielen getragen und in einer demokratischen Gesellschaft notwendig sind, d.h. durch ein zwingendes gesellschaftliches Bedürfnis gerechtfertigt sind und insbesondere in einem angemessenen Verhältnis zu dem verfolgten berechtigten Ziel stehen. ${ }^{8}{ }^{3}$ [80.] So können auch das Recht auf freie Meinungsäußerung und das Recht, sich friedlich zu versammeln, die durch die EMRK gewährleistet sind - anders als andere durch diese Konvention gewährleistete Grundrechte wie das Recht jedes Menschen auf Leben oder das Verbot der Folter oder unmenschlicher oder erniedrigender Strafe, die keinerlei Beschränkung unterliegen - keine uneingeschränkte Geltung beanspruchen, sondern müssen im Hinblick auf ihre gesellschaftliche Funktion gesehen werden. Folglich kann die Ausübung dieser Rechte Beschränkungen unterworfen werden, sofern diese Beschränkungen tatsächlich dem Gemeinwohl dienenden Zielen der Gemeinschaft entsprechen und nicht einen im Hinblick auf den mit den Beschränkungen verfolgten Zweck unverhältnismäßigen, nicht tragbaren Eingriff darstellen, der die geschützten Rechte in ihrem Wesensgehalt antastet. $\left[^{9}\right]$ [81.] Demgemäß sind die bestehenden Interessen abzuwägen, und es ist anhand sämtlicher Umstände des jeweiligen Einzelfalls festzustellen, ob das rechte Gleichgewicht zwischen diesen Interessen gewahrt worden ist. [82.] In dieser Hinsicht verfügen die zuständigen Stellen über ein weites Ermessen. Dennoch ist zu prüfen, ob die Beschränkungen, denen der innergemeinschaftliche Handel unterworfen wurde, in einem angemessenen Verhältnis zu dem berechtigten Ziel stehen, das mit ihnen verfolgt wird, hier dem Schutz der Grundrechte."

8 Vgl. in diesem Sinne Urteile von 26. Juni 1997 in der Rechtssache C-368/95, Familiapress, Slg. 1997, I-3689, Randnr. 42 = EuGRZ 1997, 344, und vom 11. Juli 2002 in der Rechtssache C-60/00, Carpenter, Slg. 2002, I-6279, Randnr. 42 = EuGRZ 2002, 332, sowie EGMR Urteil Steel u.a./Vereinigtes Königreich vom 23. September 1998, Recueil des arrêts et décisions 1998-VII, § 101.

9 Vgl. in diesem Sinne Urteile vom 8. April 1992 in der Rechtssache C-6290, Kommission/ Deutschland, Slg. 1992, I-2575, Randnr. 23, und vom 5. Oktober 1994 in der Rechtssache C-404/92 P.X/Kommission, Slg. 1994, I-4737, Randnr. 18 = EuGRZ 1995, 247. 
Die Schlussfolgerung des EuGH, im spezifischen Fall und nach Abwägung aller fallbezogenen Gegebenheiten, lautet wie folgt: »[89.] Schließlich ist, was die anderen von der Klägerin in Hinblick auf diese Versammlung genannten Möglichkeiten angeht, angesichts des weiten Ermessens, über das die Mitgliedstaaten verfügen, davon auszugehen, dass die nationalen Stellen unter solchen Umständen wie denen des Ausgangsrechtsstreits annehmen durften, dass ein schlichtes Verbot der Versammlung einen nicht hinnehmbaren Eingriff in die Grundrechte der Demonstranten, sich zu versammeln und ihre Meinung friedlich öffentlich zu äußern, dargestellt hätte. (...) [93.] Daher konnten die nationalen Stellen angesichts des weiten Ermessens, das ihnen auf diesem Gebiet zusteht, vernünftigerweise annehmen, dass das mit der Versammlung in legitimer Weise verfolgte Ziel im vorliegenden Fall nicht durch Maßnahmen erreicht werden konnte, die den innergemeinschaftlichen Handel weniger beschränkt hätten."

Im Urteil vom 14. Oktober 2004 im Fall Omega ${ }^{10}$ hat der EuGH hinsichtlich der Dienstleistungsfreiheit entschieden, dass gespieltes Töten - d.h. Schiessen mit Laserpistolen auf Sensoren-Jacken tragende Mitspieler in »Laserdrome«-Spielhalle - die Menschenwürde und die öffentliche Ordnung verletzt. Das nationale Verbot wurde hiermit bestätigt. In Punkt 34 des Urteils hat der Gerichtshof sich auf die Schlussanträge seiner Generalanwältin Christine Stix-Hackl stützend festgehalten, dass die Gemeinschaftsrechtsordnung unbestreitbar auf die Gewährleistung der Achtung der Menschenwürde als eines allgemeinen Rechtsgrundsatzes abzielt. Somit ist das Ziel, die Menschenwürde zu schützen, unzweifelhaft mit dem Gemeinschaftsrecht vereinbar, ohne dass es insoweit eine Rolle spielt, dass in Deutschland dem Grundsatz der Achtung der Menschenwürde die besondere Stellung eines selbständigen Grundrechts zukommt.

Es wird oft unterstrichen, dass es angesichts der immer weiter greifenden exekutiven Entscheidungen und Koordinierungen immer schwieriger sein wird, überzeugend abzugrenzen, was ausführendes nationales Recht ist und was dem den Einzelnen unmittelbar berührenden Gemeinschaftsrecht angehört. Man könnte auch von einer »normativen Verwirrung« sprechen, was den Grundrechtsschutz sicherlich nicht vereinfacht. In diesem Zusammenhang stellt sich folgende Frage: Ist Richtlinienkonformität ranghöchstes Normenauslegungsprinzip? Der Europäische Gerichtshof für Menschenrechte hat diese Frage schon 1996 eindeutig verneint.

\section{Der EGMR und das Cantoni-Urteil}

Im richtungweisenden Cantoni-Urteil vom 15. November $1996^{11}$ - betreffend das Apothekenmonopol und die Bestrafung wegen Verkaufs von Arzneimitteln im Super-

10 Omega Spielhallen- und Automatenaufstellungs-GmbH gegen Oberbürgermeisterin der Bundesstadt Bonn, Rs. C-36/02, Slg. 2004, I-9609; EuGRZ 2004, 639.

11 RJD 1996-V, 1614; Revue trimestrielle des droits de l'homme, 1997, 685, Anmerkung Dean Spielmann: »Principe de légalité et mise en oeuvre communautaire«; EuGRZ 1999, 193, Anmerkung Sebastian Winkler, EuGRZ 1999, 181 : »Der EGMR zum innerstaatlich und gemeinschaftsrechtlich (RL 65/65/EWG) definierten Arzneimittelbegriff beim Apothekenmonopol." 
markt auf Grund des Arzneimittelbegriffs nach innerstaatlichem und EG-Recht, d.h. der Richtlinie 65/65/EWG - hat der Gerichtshof folgendes festgehalten: »[30.] Der von der Regierung betonte Umstand, dass Art. L. 511 des code de la santé publique nahezu wortgleich der Gemeinschaftsrichtlinie 65/65 entspricht (s.o. Ziff. 12), entzieht diese Bestimmung nicht dem Anwendungsbereich des Artikels 7 der Konvention."

Ein Kommentator dieses Urteils spricht von einem »Paradigmenwechsel« und kommt zur folgenden Schlussfolgerung: »Das Urteil des EGMR in der Sache Cantoni ./. Frankreich stellt die seit langem erwartete Bestätigung für die auch gegenüber dem Gemeinschaftsrecht fortbestehende Konventionsbindung der Mitgliedstaaten dar. Das jetzt verkündete Urteil Matthews ./. Vereinigtes Königreich belegt, dass auch der neue ständige EGMR diese Rechtsprechungslinie weiterzuverfolgen gewillt ist. Das Urteil Cantoni bedeutet einen Meilenstein. (...) Der EGMR hat mit seiner Entscheidung deutlich gemacht, dass er von nun an Maßnahmen der Mitgliedstaaten auch dann auf ihre Vereinbarkeit mit der Konvention hin überprüfen wird, wenn ihre gesetzlichen Vorgaben auf Gemeinschaftsrecht beruhen sollten. Er bestätigt damit die Erkenntnis, dass die Autonomie der Gemeinschaftsrechtsordnung nur unter dem Vorbehalt der Wahrung der Konventionsgarantien besteht. $\ll^{12}$

\section{Epilog: Der EGMR und das Bosphorus-Urteil}

Das voraussichtlich letzte Wort hat der EGMR im Prinzipienurteil vom 30. Juni 2005 (Bosphorus gegen Irland) ${ }^{13}$ gesprochen - und betreffend die Beschlagnahme eines Flugzeuges im Rahmen der Sanktionen gegen die ehemalige Bundesrepublik Jugoslawien folgendes entschieden: ${ }^{14}$

- Die Beachtung des Gemeinschaftsrechts durch einen Mitgliedstaat der Gemeinschaft stellt ein sachliches, berechtigtes öffentliches Interesse i. S. von Art. 1 des Zusatzprotokolls zur EMRK ${ }^{15}$ dar. $^{16}$

12 Sebastian Winkler, EuGRZ 1999, 183, mit vgl. Hinweis auf Ress, Menschenrechte, europäisches Gemeinschaftsrecht und nationales Verfassungsrecht, in FS-G. Winkler, 1997, $897,922 \mathrm{f}$.

13 RJD 2005-VI; MenschenRechtsMagazin 2006/1, 87, aufgearbeitet von Mathias Schweitzer; NJW 2006, 197, übersetzt und bearbeitet von Dr. Jens Meyer-Ladewig und Professor Dr. Herbert Petzold. Siehe auch Christine Heer-Reißmann, »Straßburg oder Luxemburg? - Der EGMR zum Grundrechtsschutz bei Verordnungen der EG in der Rechtssache Bosphorus«, NJW, 2006, 192.

14 Leitsätze von Dr. Jens Meyer-Ladewig und Professor Dr. Herbert Petzold, ibidem.

15 Art. 1 ZP: »Jede natürliche oder juristische Person hat das Recht auf Achtung ihres Eigentums. Niemandem darf sein Eigentum entzogen werden, es sei denn, dass das öffentliche Interesse es verlangt, und nur unter den durch die allgemeinen Grundsätze des Völkerrechts vorgesehenen Bedingungen. Absatz 1 beeinträchtigt jedoch nicht das Recht des Staates, diejenigen Gesetze anzuwenden, die er für die Regelung der Benutzung des Eigentums im Einklang mit den Allgemeininteresse oder zur Sicherung der Zahlung der Steuern oder sonstigen Abgaben oder von Geldstrafen für erforderlich hält.«

16 Ziff. 150. 
- Die EMRK verbietet es den Vertragsstaaten nicht, Hoheitsbefugnisse auf eine internationale oder supranationale Organisation zu übertragen. Doch ist jeder Vertragsstaat nach Art. 1 EMRK $^{17}$ für alle Handlungen und Unterlassungen seiner Organe verantwortlich, und das auch dann, wenn es dabei um die Erfüllung völkerrechtlicher Verpflichtungen geht: Der Staat bleibt nach der Konvention verantwortlich, wenn er vertraglichen Verpflichtungen nachkommt, die er nach In-Kraft-Treten der EMRK eingegangen ist. ${ }^{18}$

- Staatliches Handeln in Erfüllung solcher Verpflichtungen im Rahmen einer internationalen Organisation ist gerechtfertigt, wenn die Organisation die Grundrechte schützt und dieser Schutz wenigstens als »gleichwertig « zu dem der EMRK anzusehen ist, d.h. als »vergleichbar«, nicht als »identisch«. In diesem Fall besteht eine Vermutung, dass der Staat sich den Anforderungen der EMRK nicht entzogen hat. Die Vermutung kann jedoch widerlegt werden, wenn der gewährte Grundrechtsschutz offensichtlich unzureichend ist. ${ }^{19}$

- Der Schutz der Grundrechte im Gemeinschaftsrecht ist dem der EMRK »gleichwertig«, und auch der Schutz der Grundrechte der Beschwerdeführerin war nicht offensichtlich unzureichend. ${ }^{20}$

Nach Maßgabe dieser Voraussetzungen hat die Beschlagnahme des Flugzeugs der Beschwerdeführer Art. 1 Zusatzprotokoll zur EMRK nicht verletzt. ${ }^{21}$ Weitere Fälle werden dem Europäischen Gerichtshof für Menschenrechte sicherlich die Gelegenheit bieten, eine eventuelle Widerlegung der Vermutungsregel zu prüfen. Wir sind also kein Gericht im »Wartestand. «Wir sind ein Gericht im »prüfenden Stand «.

Ein letztes Wort bezüglich der EU-Charta der Grundrechte: Zu Recht weist Burkhard Hirsch - wenn auch unter Vorbehalt - darauf hin, dass die Charta angesichts ihrer feierlichen Verkündigung auch ohne formelle Rechtskraft als gemeinsamer Grundstock der Verfassungsrechte der EU-Mitglieder in der Rechtsprechung des EuGH beachtet wird. Von einer Globalisierung im europäischen Grundrechtsschutz hatte Johan Callewaert (2003) in einem viel beachteten Artikel in der Europäischen Grundrechtezeitschrift gesprochen. ${ }^{22}$ Der Untertitel lautete: »Bestandsaufnahme einer Harmonisierung auf halbem Weg.« Bezüglich der Charta der Grundrechte, die leider nicht in Kraft getreten ist, hatte der Autor optimistisch bemerkt: »Die Charta hat die Interdependenz besiegelt, die zwischen der EMRK und dem Gemeinschaftsrecht längst zur Realität geworden war, und sie auf das ganze Recht der Union ausgeweitet. Was ohnehin schon Tatsache war, wurde nun formell bestätigt und beurkundet: Keine der beiden europäischen Grundrechtsordnungen, weder die EMRK noch die EU-Grundrechtsordnung, kann noch völlig autonom, d.h. ohne Rückkoppelung mit der anderen,

17 Art. 1 EMRK : »Die Hohen Vertragsparteien sichern allen ihrer Hoheitsgewalt unterstehenden Personen die in Abschnitt I bestimmten Rechte und Freiheiten zu.«

18 Ziff. 152-154.

19 Ziff. 155 und 156.

20 Ziff. 165 und 166.

21 Ziff. 167.

22 Johan Callewaert, »Die EMRK und die EU-Grundrechtecharta. Bestandsaufnahme einer Harmonisierung auf halbem Weg«, EuGRZ 2003, S. 198 ff. 
in rechtlich befriedigender Weise funktionieren. Diese Interdependenz entspricht nichts anderem als einer Art Globalisierung im europäischen Grundrechtsschutz, die es zu begrüßen gilt und von der es ohnehin wohl kein Zurück mehr geben dürfte. In der Tat wird es in dem Maß, in dem die EU in Bereiche vorstößt, die bis vor kurzem noch ausschließlich von den Mitgliedstaaten betreut wurden, zunehmend schwierig zu begründen, warum die gleichen Grundrechte als Folge der EU-Zuständigkeit plötzlich anders auszufüllen wären. Wie zutreffend bemerkt wurde, sind es die gleichen Grundrechtsträger - die Bürger -, die von beiden Grundrechtsordnungen erfasst werden und wenig Verständnis für Inkohärenzen auf diesem Gebiet aufbringen dürften. $\left.{ }^{23}\right]$ Die Antwort darauf ist die Schaffung eines europäischen Grundrechteraums als Teil des europäischen Verfassungsraums, auf den Rodriguez Iglesias in seiner Straßburger Rede verwies. $\left[{ }^{24}\right]$ Mit der Charta wurde dazu ein wichtiger Beitrag geleistet. Um wirksam zu werden, soll nun allerdings die Interdependenz mit der EMRK, die die Charta im Bereich der Texte besiegelt hat, eine institutionelle und verfahrensrechtliche Fortsetzung erhalten. Den Grundstein dazu bildet der Beitritt der EU zur EMRK, der damit neben der Charta zu einem gemeinsamen Anliegen aller europäischen Staaten sowie der EU selbst geworden ist.« - Ich schließe mich diesen Worten an.

Ein effektiver Grundrechtsschutz ist ohne die unermüdliche Tätigkeit von engagierten Rechtsanwälten nicht möglich. Aber auch der Politiker ist gefordert, seine Tätigkeit stets am Maßstab der Menschenrechte zu prüfen. Und wie Burkhard Hirsch immer wieder unter Beweis stellt, sind ebenfalls große wissenschaftliche Kenntnisse erforderlich, um in einer von der Globalisierung und für den Bürger normativ verwirrenden Umgebung die angesprochene Rückkopplung der verschiedenen Grundrechtsordnungen zu bewerkstelligen.

23 W. Hoffmann-Riem, »Kohärenz der Anwendung europäischer und nationaler Grundrechte«, EuGRZ 2002, S. $473 \mathrm{ff}$.

24 »Die beiden [europäischen] Gerichtshöfe teilen eine existenzielle Verpflichtung gegenüber den Grundwerten, die zum gemeinsamen Erbe Europas gehören und deren Grundlage Demokratie und Menschenrechte sind. So gesehen, tragen sie - zusammen mit den nationalen Höchst- und Verfassungsgerichten - zum Entstehen eines, wie man es hat nennen können, >europäischen Verfassungsraumes< bei.« (Übers., EuGRZ 2002, S. 206). 